\title{
Viabilidad de repositorios de biomedicina y ciencias de la salud en la Comunidad Valenciana
}

\author{
Por María-Francisca Abad-García, Aurora González-Teruel, Celeste Martínez-Catalán y Francesc \\ Giménez-Martínez
}

Resumen: Se estudian las condiciones de autoarchivo de 3.495 trabajos publicados en 109 revistas que recogen el $50 \%$ de la producción científica de la Comunidad Valenciana en biomedicina y ciencias de la salud difundida en las bases de datos ISI (período 2000-2004). Se analiza el autoarchivado desde la perspectiva de la viabilidad de implantación de repositorios institucionales y temáticos de acceso abierto. La información se ha obtenido de la base de datos Romeo disponible en la web Sherpa. Las condiciones de autoarchivo son favorables para la implantación de repositorios institucionales ya que el 56,8\% de los trabajos sería depositable inmediatamente, cifra que ascendería al $72 \%$ al considerar los depositables tras un período de embargo, y al $87 \%$ al incluir los trabajos publicados en revistas accesibles a texto completo. La viabilidad para establecer un repositorio temático de biomedicina y ciencias de la salud de la Comunidad Valenciana es cuestionable debido a que la mayoría de las editoriales condicionan el permiso para el depósito de trabajos a la existencia de un mandato de las agencias estatales y/o autonómicas responsables de la financiación de la investigación, algo inexistente en este momento.

Palabras clave: Acceso abierto, Repositorios institucionales, Repositorios temáticos, Biomedicina y ciencias de la salud, Comunidad Valenciana.

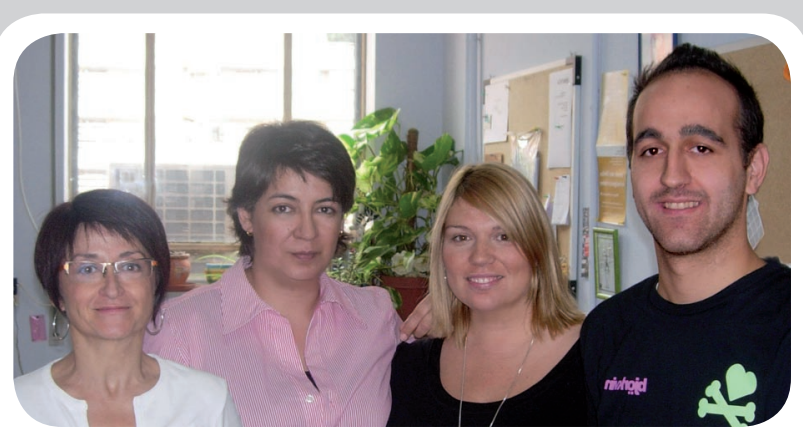

Maria-Francisca Abad-García es doctora en medicina y profesora titular del área de biblioteconomía y documentación de la Universidad de Valencia. Desde 2002 es la directora técnica de la Biblioteca Médica Virtual del Colegio Oficial de Médicos de Valencia.

Aurora González-Teruel es doctora en geografía e historia y profesora titular del área de biblioteconomía y documentación de la Universidad de Valencia. Desde 2002 es la coordinadora de la Biblioteca Médica Virtual del Colegio Oficial de Médicos de Valencia.

Celeste Martínez-Catalán es documentalista de la Biblioteca Médica Virtual del Colegio Oficial de Médicos de Valencia.

Francesc Giménez-Martínez es diplomado en biblioteconomía y documentación y es becario de la Biblioteca Médica Virtual del Colegio Oficial de Médicos de Valencia.

\section{Title: Viability of establishing institutional and biomedical/health sciences repositories in Va- lencia}

Abstract: Self-archiving was studied in 3,495 papers in 109 core journals that published 50\% of Valencia's scientific production in biomedical and health sciences included in the 2000-2004 ISI databases. The effectiveness of self-archiving is analyzed with respect to the feasibility of launching open access institutional and subject-specific repositories. Data were obtained from the Sherpa/Romeo database. Self-archiving is favourable for the implementation of institutional repositories because $56.8 \%$ of papers could be immediately deposited, a percentage that increases to $72 \%$ after a period of embargo and to $87 \%$ when papers published in full text journals are included. The feasibility of establishing biomedical and health science repositories for the Valencian Community is questionable, due to the fact that most publishers will not grant permission for inclusion in a repository unless mandated by the research funding agency, a practice lacking in our country at present.

Keywords: Open access, Institutional repositories, Thematic repositories, Subject repositories, Biomedicine and health sciences, Valencian Community, Spain.

Abad-García, María-Francisca; González-Teruel, Aurora; Martínez-Catalán, Celeste; Giménez-Martínez, Francesc. "Viabilidad de repositorios de biomedicina y ciencias de la salud en la Comunidad Valenciana". En: El profesional de la información, 2008, marzo-abril, v. 17, n. 2, pp. 165-173.

DOI: 10.3145/epi.2008.mar.05

\section{Introducción}

Un autor puede proporcionar acceso abierto a sus trabajos depositándolos en un repositorio o depósito de $e$-prints (autoarchivo) o publicando en una revista de acceso abierto $^{1}$. Los primeros son servidores que almacenan recursos digitales (textuales, de imagen o sonido) como medio para garantizar su permanencia, preservación, difusión y libre acceso. Pueden ser de pre-prints si incluyen trabajos no sometidos a revisión 
por pares (comunicaciones, artículos no evaluados o en fase de evaluación, informes, tesis, etc.) o post-prints si han superado este proceso $^{2}$.

Pueden ser institucionales o temáticos. Aunque ambos cumplen el mismo objetivo, los primeros si están completos proporcionan una imagen de la experiencia investigadora de la organización y sirven como archivo para su memoria institucional, mientras que los temáticos reúnen los resultados de la investigaciones en un área temática (y en ocasiones también geográfica) ${ }^{3}$, implicando directa o indirectamente múltiples instituciones.

El número de repositorios aumenta progresivamente a medida que las instituciones, sociedades científicas, investigadores y la sociedad en general toman conciencia de la trascendencia del acceso abierto para el progreso de la ciencia. El directorio de depósitos de acceso abierto OpenDOAR de la Universidad de Nottingham ${ }^{4}$ incluye 1.013 , de los que 820 se han clasificado en la categoría de institucionales. De ellos, 15 corresponden a instituciones españolas, siendo el repositorio de la Universidad de Alicante ${ }^{5}$ el único de una institución de la Comunidad Valenciana.

En biomedicina, con un volumen de información que supera el $45 \%$ de la publicada en el conjunto de todas las áreas 6 , la opción de los repositorios temáticos es muy interesante, aunque las experiencias son escasas. De hecho en el OpenDOAR sólo hay 76 depósitos que tratan las materias health and medicine. De éstos, 12 son temáticos, entre los que se encuentran como ejemplos emblemáticos PubMed Central y $U K$ PubMed Central (Ukpmc). El primero fue creado en el año 2000 por los National Institutes of Health (NIH) de EUA y operado por The National Center for Biotechnology Information $(\mathrm{NCBI})^{7}$, mientras que Ukpmc es el mirror de PubMed Central creado en 2007 en el Reino Unido y promovido por nueve importantes instituciones británicas"; 15 son del tipo "agregados" o repositorios que agrupan revistas de acceso abierto, de los que 12 son las distintas "ubicaciones geográficas" de Scielo entre las que está Scielo España $; 47$ son institucionales (16 exclusivos de medicina y salud y 30 de varias temáticas que incluyen la medicina) y finalmente dos son gubernamentales (uno monográfico y otro multidisciplinar).

De acuerdo con esto, la situación española en biomedicina no es boyante, aunque puede cambiar próximamente ya que varias bibliotecas virtuales de salud de las administraciones sanitarias autonómicas se plantean la creación de repositorios ${ }^{10}$.

La Comunidad Valenciana ocupa el cuarto lugar en el ranking español de producción científica en biomedicina y ciencias de la salud ${ }^{11}$ y sin embargo no existen iniciativas conocidas para la implantación de depósitos, algo que representaría un indudable beneficio para los investigadores con el fin de lograr la libre circulación de la ciencia y mejorar la visibilidad de la investigación biomédica de esta comunidad autónoma.

Los beneficios potenciales de un repositorio están condicionados por las dificultades de su puesta en marcha y por el reto que supone su aceptación y uso por parte de los autores de los trabajos. En su desarrollo, lo primero a considerar es si los autores pueden depositar sus trabajos. Las revistas que no son de acceso abierto requieren como condición que los autores renuncien a sus derechos de propiedad intelectual de forma permanente a favor de la editorial. Así, los derechos de reproducción, copia y difusión quedan en poder de la editorial de la revista, y el autor podrá disponer de su trabajo sólo en los términos que la editorial establezca, lo que también afecta al autoarchivo.

Por ello parece lógico que el primer paso para promover la creación de estos repositorios sea el estudio de las condiciones de autoarchivo de los trabajos que constituirían su población diana. Conocer esta información y valorar sus consecuencias para cada tipo de repositorio, permitiría elaborar un análisis de situación del que pudieran derivarse recomendaciones en uno $\mathrm{u}$ otro sentido.

\section{"El primer paso para promover la creación de un repositorio es conocer las condiciones de autoarchivo de los trabajos que lo constituirían"}

\section{Objetivo}

En este artículo nos propusimos analizar las posibilidades de autoarchivo para documentos post-print en biomedicina en repositorios tanto temáticos como institucionales de la Comunidad Valenciana.

Para ello se han estudiado las condiciones que establecen las editoriales de las revistas indizadas en el Science Citation Index y en el Social Science Citation Index en las que han publicado trabajos de biomedicina y ciencias de la salud los científicos de dicha Comunidad durante el período 2000-2004.

\section{Material y método}

El material utilizado son los 3.495 trabajos publicados en 109 revistas que recogen el 50\% de la producción científica (revistas nucleares) de la Comunidad Valenciana en biomedicina y ciencias de la salud difun- 
dida en las bases de datos ISI (2000-2004) $)^{12}$. Para cada una de las publicaciones se ha obtenido información sobre:

a. Número de trabajos publicados (datos procedentes del estudio antes mencionado).

b. Editorial, tipo de acceso a los contenidos de la revista (por suscripción o acceso gratuito al texto completo con o sin embargo) y vinculación al movimiento de acceso abierto (datos obtenidos mediante consulta directa de la información editorial presente en la web de la revista).

c. Posibilidades de autoarchivo de los trabajos según:

- tipo de documento (pre-print o post-print);

- momento del autoarchivo (tras la aceptación del trabajo, en el momento de la publicación o después de un período de embargo);

- tipo de depósito donde puede realizarse el autoarchivo (web personal, repositorio institucional o temático);

- versión para el depósito (versión del autor o pdf de la versión publicada en la revista);

- otras condiciones y restricciones para el autoarchivo (mención de copyright y enlace a fuente de publicación del trabajo); y por último,

- acceso abierto a los contenidos de la revista mediante el pago de tasas por el autor (author pays).

Para conocer las condiciones arriba especificadas se ha consultado la web del proyecto Sherpa/Romeo ${ }^{13}$. Se trata de un sitio creado con el apoyo del JISC (Joint Information Systems Committee) y del Wellcome Trust que aloja, entre otros recursos, una base de datos resultante del proyecto Romeo (Rights metadata for open archiving) en la que se especifica la información relativa a los permisos de autoarchivo de los trabajos, que ha sido extraída de las condiciones de transferencia de los derechos de autor especificadas por las editoriales.
Para el análisis del tipo de documento que puede archivarse, se ha utilizado la clasificación de Sherpal Romeo según la cual a las editoriales y a las revistas se les asigna uno de los siguientes colores: blanco si no permite el autoarchivo de ninguna versión; amarillo en el caso sólo del pre-print; azul cuando es el post-print del autor, y verde si es posible el autoarchivo de las dos versiones pre y post-print.

\section{Resultados}

\section{Características de la población estudiada}

En las 109 revistas se han publicado 3.594 trabajos, 1.050 en revistas españolas $(29,2 \%)$ y $2.544(71,8 \%)$ en internacionales. Están editadas por 39 editoriales de las que 19 son comerciales, 17 sociedades científicas y 3 universidades. Las editoriales comerciales agrupan el $82,9 \%$ de los trabajos publicados mientras que el $17,1 \%$ restante lo publican sociedades científicas y universidades (tabla 1).

De entre las editoriales comerciales (tabla 2), Elsevier es la líder con 37 revistas en las que se publican el $47 \%$ de los trabajos de este sector editorial y el $38,8 \%$ de los que constituyen nuestra población de estudio. Incluye a cinco revistas españolas editadas anteriormente por Doyma donde se publicaron 554 trabajos $(15,4 \%$ del total de los trabajos nucleares).

\section{Tipo de acceso a los contenidos de las revistas}

El acceso al texto completo del documento varía mucho según sean revistas nacionales o internacionales (tabla 3). Así, mientras el 50,7\% de los trabajos que se publican en revistas españolas son accesibles al texto completo, esto sólo sucede en el 1,9\% de los materiales publicados en las internacionales. Si también se consideran las accesibles a texto completo tras un período de embargo, estos porcentajes varían siendo el $67,5 \%$ para las nacionales y el $31,5 \%$ para las internacionales.

El período de embargo varía habitualmente entre un año (15 revistas), o seis meses (10 revistas). Únicamente en un caso se trata sólo de tres meses y en

\begin{tabular}{|c|c|c|c|c|c|c|c|c|c|c|c|c|}
\hline & \multicolumn{2}{|c|}{$\begin{array}{l}\text { Revistas } \\
\text { españolas }\end{array}$} & \multicolumn{2}{|c|}{$\begin{array}{l}\text { Trabajos } \\
\text { publicados } \\
2000-2004\end{array}$} & \multicolumn{2}{|c|}{$\begin{array}{c}\text { Revistas } \\
\text { internaciona- } \\
\text { les }\end{array}$} & \multicolumn{2}{|c|}{$\begin{array}{l}\text { Trabajos } \\
\text { publicados } \\
2000-2004\end{array}$} & \multicolumn{2}{|c|}{$\begin{array}{c}\text { Total } \\
\text { revistas }\end{array}$} & \multicolumn{2}{|c|}{$\begin{array}{c}\text { Total } \\
\text { trabajos }\end{array}$} \\
\hline & No & $\%$ & No & $\%$ & No & $\%$ & No & $\%$ & No & $\%$ & No & $\%$ \\
\hline E. comercial & 10 & 79,9 & 925 & 88,1 & 76 & 76,2 & 2.054 & 80,7 & 86 & 78,9 & 2.979 & 82,9 \\
\hline $\begin{array}{l}\text { Sociedad } \\
\text { científica }\end{array}$ & 1 & 7,7 & 16 & 1,5 & 19 & 19,8 & 471 & 18,5 & 20 & 18,3 & 487 & 13,6 \\
\hline Universidad & 2 & 15,4 & 109 & 10,4 & 1 & 1,1 & 19 & 0,7 & 3 & 2,8 & 128 & 3,6 \\
\hline Total & 13 & 100,0 & 1.050 & 100,0 & 96 & 100,0 & 2.544 & 100,0 & 109 & 100,0 & 3.594 & 100,0 \\
\hline
\end{tabular}

Tabla 1. Distribución de los trabajos publicados por tipo de editorial y país de edición de las revistas 
otro, la Revista española de enfermedades digestivas, es imposible determinar si existe un embargo de más de un año o se trata de un retraso en la actualización de su incorporación a Scielo.

\begin{tabular}{|c|c|c|c|}
\hline Editorial & $\begin{array}{c}\text { No } \\
\text { revistas }\end{array}$ & $\begin{array}{c}\text { No } \\
\text { trabajos }\end{array}$ & $\begin{array}{c}\% \\
\text { trabajos }\end{array}$ \\
\hline Elsevier* & 37 & 1.408 & 47,29 \\
\hline Blackwell & 11 & 258 & 8,68 \\
\hline Springer & 9 & 179 & 6,01 \\
\hline $\begin{array}{l}\text { Lippincott } \\
\text { Williams \& } \\
\text { Wilkins }\end{array}$ & 5 & 194 & 6,52 \\
\hline $\begin{array}{l}\text { John Wiley \& } \\
\text { Sons }\end{array}$ & 4 & 123 & 4,12 \\
\hline $\begin{array}{l}\text { Oxford } \\
\text { University Press }\end{array}$ & 4 & 170 & 5,70 \\
\hline $\begin{array}{l}\text { Nature } \\
\text { Publishing }\end{array}$ & 3 & 75 & 2,51 \\
\hline Ars $X X I I$ & 2 & 68 & 2,28 \\
\hline Aran & 1 & 67 & 2,24 \\
\hline Aula Médica & 1 & 77 & 2,58 \\
\hline BMJ Publishing & 1 & 34 & 1,14 \\
\hline Ferrata Sorli & 1 & 48 & 1,61 \\
\hline George Thieme & 1 & 20 & 0,67 \\
\hline $\begin{array}{l}\text { Harvey Whitney } \\
\text { Books Company }\end{array}$ & 1 & 15 & 0,50 \\
\hline IOS Press & 1 & 14 & 0,47 \\
\hline Portland Press & 1 & 17 & 0,57 \\
\hline Slack & 1 & 27 & 0,91 \\
\hline Taylor \& Francis & 1 & 26 & 0,87 \\
\hline Viguera & 1 & 159 & 5,33 \\
\hline Total & 86 & 2.979 & 100,00 \\
\hline
\end{tabular}

Tabla 2. Distribución de las revistas y de los trabajos según editorial comercial

\section{Vinculación explícita de las revistas con el movi- miento open access}

Ninguna de las 35 revistas que permiten el acceso libre a sus contenidos, bien inmediato o tras un período de embargo, está vinculada a este movimiento. Así, pese a que dos de ellas se integran en Scielo, los derechos de propiedad intelectual quedan en poder de la revista y no realizan la cesión de uso de los contenidos que caracteriza a las publicaciones de acceso abierto. Lo mismo ocurre con los tres títulos (Biology \& reproduction, Chest y el International journal of developmental biology) que están relacionadas a los Washington principles for free access to science ${ }^{14}$ pues aunque permiten el libre acceso por los mismos motivos que las anteriores, no son de acceso abierto.

\section{Presencia de información sobre las editoriales y revistas en la web del proyecto Sherpa/Romeo}

Se recogen las condiciones de autoarchivo de 88 revistas (publicadas por 24 editoriales). No hay información sobre 21 revistas (correspondientes a 15 editoriales) de las que 12 son españolas. La única publicación española de la que hay información es Archivos de bronconeumología editada por Doyma-Elsevier, pero no se ha tenido en cuenta hasta verificar sus condiciones con la editorial.

\section{Condiciones de autoarchivo de las revistas}

Sherpa/Romeo proporciona información sobre las condiciones de autoarchivo de 24 de las 39 editoriales. De éstas, la mayoría permite el autoarchivo de la versión postprint (editoriales verdes y azules), seguidas de las editoriales blancas que no permiten ningún tipo de depósito (tabla 5).

La repercusión de estas políticas editoriales se aprecia mejor en la tabla 6 donde se ofrecen los datos sobre las condiciones de autoarchivo de las revistas y el número de artículos según el color de la editorial. Aquellas revistas para las que no existe información en la web de Sherpa se han clasificado de acuerdo con el tipo de acceso a sus contenidos. Las cinco revistas españolas editadas por Doyma-Elsevier se han clasificado

\begin{tabular}{|l|r|r|r|r|r|r|r|r|r|}
\hline \multicolumn{1}{|c|}{ País de edición } & \multicolumn{3}{|c|}{ Españolas } & \multicolumn{2}{c|}{ Internacionales } & \multicolumn{3}{c|}{ Total } \\
\hline & $\begin{array}{c}\text { No } \\
\text { rev }\end{array}$ & $\begin{array}{c}\text { No } \\
\text { trab }\end{array}$ & $\begin{array}{c}\text { \% } \\
\text { trab }\end{array}$ & $\begin{array}{l}\text { No } \\
\text { rev }\end{array}$ & $\begin{array}{c}\text { No } \\
\text { trab }\end{array}$ & $\begin{array}{c}\text { \% } \\
\text { trab }\end{array}$ & $\begin{array}{l}\text { No } \\
\text { rev }\end{array}$ & $\begin{array}{c}\text { No } \\
\text { trab }\end{array}$ & $\begin{array}{c}\text { \% } \\
\text { trab }\end{array}$ \\
\hline Acceso libre al texto completo & 7 & 532 & 50,7 & 1 & 48 & 1,9 & 8 & 580 & 16,1 \\
\hline $\begin{array}{l}\text { Acceso al texto completo con } \\
\text { embargo }\end{array}$ & 3 & 176 & 16,8 & 23 & 754 & 29,6 & 26 & 930 & 25,9 \\
\hline Acceso al texto por suscripción & 3 & 342 & 32,6 & 72 & 1.742 & 68,5 & 75 & 2.084 & 58,0 \\
\hline Total & 13 & 1.050 & 100,0 & 96 & 2.544 & 100,0 & 109 & 3.994 & 100,0 \\
\hline
\end{tabular}

Tabla 3. Distribución del tipo de acceso a los trabajos según sean revistas nacionales o internacionales 
con el color azul (permite depósito de la versión postprint pero no pre-print), de acuerdo con la información proporcionada al consultar a la editorial, lo que las diferencia del resto de las revistas de Elsevier clasificadas con el color verde.

El 57,4\% de los trabajos pueden ser archivados en acceso abierto (revistas verdes o azules) mientras que sólo un $8,4 \%$ no permite ningún tipo de autoarchivo (revistas blancas).

De un $18,9 \%$ de trabajos no se conocen sus condiciones de autoarchivo, de los que un 3,8\% corresponde a los publicados en revistas de suscripción, un 9,3\% ac- cesibles a texto completo y un 5,6\% accesibles a texto completo tras un período de embargo.

\section{Condiciones y restricciones del autoarchivo}

Las revistas publicadas por editoriales verdes y azules permiten el depósito inmediato de trabajos tanto en una web personal como en un repositorio institucional, si bien no siempre se especifica si el depósito puede hacerse tras la aceptación del artículo o tras su publicación, motivo por el que este dato no se ha tabulado (tabla 7). Las editoriales amarillas, aunque a priori no permiten el depósito de la versión post-print, en el apartado de condiciones y restricciones de autoarchivo

\begin{tabular}{|l|r|r|r|r|r|r|r|r|r|}
\hline \multicolumn{1}{|c|}{ País de edición } & \multicolumn{3}{|c|}{ Españolas } & \multicolumn{2}{c|}{ Internacionales } & \multicolumn{3}{c|}{ Total } \\
\hline & $\begin{array}{c}\text { No } \\
\text { rev }\end{array}$ & $\begin{array}{c}\text { No } \\
\text { trab }\end{array}$ & $\begin{array}{c}\text { \% } \\
\text { trab }\end{array}$ & $\begin{array}{c}\text { No } \\
\text { rev }\end{array}$ & $\begin{array}{c}\text { No } \\
\text { trab }\end{array}$ & $\begin{array}{c}\text { \% } \\
\text { trab }\end{array}$ & $\begin{array}{c}\text { No } \\
\text { rev }\end{array}$ & $\begin{array}{c}\text { No } \\
\text { trab }\end{array}$ & $\begin{array}{c}\text { \% } \\
\text { trab }\end{array}$ \\
\hline Presencia en Sherpa/Romeo & 1 & 69 & 6,6 & 87 & 2.359 & 90,6 & 88 & 2.428 & 67,6 \\
\hline Ausencia en Sherpa/Romeo & 12 & 981 & 93,4 & 9 & 185 & 9,4 & 21 & 1.166 & 32,4 \\
\hline Total & 13 & 1.050 & 100,0 & 96 & 2.544 & 100,0 & 109 & 3.594 & 100,0 \\
\hline
\end{tabular}

Tabla 4. Distribución de las revistas según la presencia Sherpa/Romeo de información sobre sus condiciones para el autoarchivo de trabajos

\begin{tabular}{|l|c|c|c|c|c|c|}
\hline Tipo editorial & Verde & Azul & Amarillo & Blanco & No consta & total \\
\hline Comercial & $5^{*}$ & $1^{*}$ & 4 & 3 & 7 & 19 \\
\hline Soc. científica & 4 & 3 & - & 3 & 7 & 17 \\
\hline Universidad & - & - & 1 & - & 2 & 3 \\
\hline Total & 9 & 4 & 5 & 6 & 15 & 39 \\
\hline
\end{tabular}

Tabla 5. Distribución de las editoriales por tipo y color

* Las revistas de Elsevier se han contabilizado según las posibilidades de autoarchivo de sus revistas internacionales y las que permite para las revistas españolas antes editadas por Doyma.

\begin{tabular}{|l|r|r|r|r|r|r|r|r|r|}
\hline \multirow{2}{*}{ Color } & \multicolumn{3}{|c|}{ Nacionales } & \multicolumn{2}{c|}{ Internacionales } & \multicolumn{3}{c|}{ Total } \\
\cline { 2 - 11 } & $\begin{array}{c}\text { No } \\
\text { rev }\end{array}$ & $\begin{array}{c}\text { No } \\
\text { trab }\end{array}$ & $\begin{array}{c}\text { \% } \\
\text { trab }\end{array}$ & $\begin{array}{c}\text { No } \\
\text { rev }\end{array}$ & $\begin{array}{c}\text { No } \\
\text { trab }\end{array}$ & $\begin{array}{c}\text { \% } \\
\text { trab }\end{array}$ & $\begin{array}{c}\text { No } \\
\text { rev }\end{array}$ & $\begin{array}{c}\text { No } \\
\text { trab }\end{array}$ & $\begin{array}{c}\text { \% } \\
\text { trab }\end{array}$ \\
\hline Verde & - & - & - & 50 & 1.310 & 51,5 & 51 & 1.329 & 37,0 \\
\hline Azul & 5 & 554 & 52,8 & 5 & 179 & 7,0 & 10 & 733 & 20,4 \\
\hline Amarillo & - & - & - & 21 & 567 & 22,3 & 20 & 548 & 15,2 \\
\hline Blanco & - & - & - & 11 & 303 & 11,9 & 11 & 303 & 8,4 \\
\hline Suscripción * & 1 & 32 & 3,0 & 6 & 103 & 4,0 & 7 & 135 & 3,8 \\
\hline Texto completo * & 4 & 288 & 27,4 & 1 & 48 & 1,9 & 5 & 336 & 9,3 \\
\hline $\begin{array}{l}\text { Texto completo con } \\
\text { embargo* }\end{array}$ & 3 & 176 & 16,8 & 2 & 34 & 1,3 & 5 & 210 & 5,8 \\
\hline Total & 13 & 1.050 & 100,0 & 96 & 2.544 & 100,0 & 109 & 3.594 & 100,0 \\
\hline
\end{tabular}

Tabla 6. Distribución de las revistas según los colores asignados por Sherpa/Romeo de acuerdo con las condiciones para el autoarchivo de sus trabajos

*: tipo de acceso de las revistas que no constan en Sherpa/Romeo. 
especifican que sí es posible en un repositorio institucional, siempre y cuando se respete un período de embargo. Finalmente, las editoriales blancas, salvo casos excepcionales que lo permiten bajo petición, suelen restringir el depósito.

En lo tocante al depósito de la versión post-print de trabajos en repositorios temáticos, los criterios no son uniformes. Lo más frecuente es que no se especifique tal posibilidad y cuando esto sucede el permiso para el depósito suele: mencionar explícitamente al reposito-

\section{"Algunas editoriales ofrecen publicar en acceso abierto si el autor (o su institución) pagan cierta cantidad de dinero"}

rio PubMed Central, exigir un período de embargo y condicionar el permiso al requisito de obligatoriedad del depósito establecido por la entidad que financia la investigación.

\begin{tabular}{|c|c|c|c|c|}
\hline Color & $\begin{array}{c}\text { No de } \\
\text { editoriales }\end{array}$ & $\begin{array}{l}\text { Depósito en web personal } \\
\text { o repositorio institucional }\end{array}$ & $\begin{array}{c}\text { Depósito en repositorio } \\
\text { temático }\end{array}$ & Editorial \\
\hline \multirow[t]{3}{*}{ Verde } & \multirow[t]{3}{*}{9} & \multirow{3}{*}{$\begin{array}{l}\text { Depósito inmediato una vez } \\
\text { recibidas las correcciones } \\
\text { o en el momento de } \\
\text { publicación del trabajo }\end{array}$} & $\begin{array}{l}3 \text { editoriales no mencionan } \\
\text { la posibilidad }\end{array}$ & $\begin{array}{l}\text { Elsevier } \\
\text { John Wiley }\end{array}$ \\
\hline & & & $\begin{array}{l}2 \text { editoriales lo permiten } \\
\text { si así lo exige la agencia } \\
\text { financiadora }\end{array}$ & $\begin{array}{l}\text { IOS Press } \\
\text { Spinger }\end{array}$ \\
\hline & & & $\begin{array}{l}3 \text { editoriales imponen un } \\
\text { período de embargo para } \\
\text { el depósito y mencionan } \\
\text { concretamente PubMed } \\
\text { Central }\end{array}$ & $\begin{array}{l}\text { American Institute } \\
\text { of Physics } \\
\text { Biophysical Society } \\
\text { MBJ Publishing } \\
\text { Group }\end{array}$ \\
\hline \multirow[t]{3}{*}{ Azul } & \multirow[t]{3}{*}{4} & \multirow{3}{*}{$\begin{array}{l}\text { Depósito inmediato una vez } \\
\text { recibidas las correcciones } \\
\text { o en el momento de } \\
\text { publicación del trabajo }\end{array}$} & $\begin{array}{l}1 \text { editorial no menciona la } \\
\text { posibilidad }\end{array}$ & Doyma-Elsevier \\
\hline & & & $\begin{array}{l}1 \text { editorial lo permite si } \\
\text { así lo exige la agencia } \\
\text { financiadora }\end{array}$ & $\begin{array}{l}\text { American Society } \\
\text { for Microbiology }\end{array}$ \\
\hline & & & $\begin{array}{l}2 \text { editoriales imponen un } \\
\text { período de embargo para } \\
\text { el depósito y mencionan } \\
\text { concretamente PubMed } \\
\text { Central }\end{array}$ & $\begin{array}{l}\text { American Society of } \\
\text { Hematology } \\
\text { Society for Neuro- } \\
\text { sciencies }\end{array}$ \\
\hline \multirow[t]{2}{*}{ Amarillo } & \multirow[t]{2}{*}{5} & \multirow[t]{2}{*}{$\begin{array}{l}\text { Depósito con embargo de } \\
\text { entre } 6 \text { y } 12 \text { meses }\end{array}$} & $\begin{array}{l}3 \text { editoriales no mencionan } \\
\text { la posibilidad }\end{array}$ & $\begin{array}{l}\text { Blackwell } \\
\text { Oxford } \\
\text { Taylor \& Francis }\end{array}$ \\
\hline & & & $\begin{array}{l}2 \text { editoriales permiten } \\
\text { el depósito y nombran } \\
\text { específicamente PubMed } \\
\text { Central cumpliendo el } \\
\text { período de embargo }\end{array}$ & $\begin{array}{l}\text { Nature Publishing } \\
\text { University of Chi- } \\
\text { cago Press }\end{array}$ \\
\hline \multirow[t]{2}{*}{ Blanco } & \multirow[t]{2}{*}{6} & $\begin{array}{l}1 \text { editorial no permite } \\
\text { ningún tipo de autoarchivo } \\
1 \text { editorial con condiciones } \\
\text { dudosas } \\
1 \text { requiere permiso } \\
\text { específico de la editorial }\end{array}$ & $\begin{array}{l}3 \text { editoriales no permiten el } \\
\text { depósito }\end{array}$ & $\begin{array}{l}\text { Lippincott } \\
\text { Faseb } \\
\text { Portland Press }\end{array}$ \\
\hline & & $\begin{array}{l}1 \text { establece embargo de } 6 \\
\text { meses } \\
2 \text { editoriales sólo permiten } \\
\text { depósito en repositorio } \\
\text { temático si es condición de } \\
\text { la agencia financiadora }\end{array}$ & $\begin{array}{l}3 \text { editoriales permiten } \\
\text { el depósito en PubMed } \\
\text { Central o repositorio } \\
\text { temático correspondiente } \\
\text { según determine la agencia } \\
\text { financiadora }\end{array}$ & \\
\hline
\end{tabular}

Tabla 7. Distribución según el momento y tipo de repositorio en el que los distintos tipos de editoriales, clasificadas por colores, permiten el depósito de la versión post print de los trabajos 
Otra condición a tener en cuenta es que la versión que debe archivarse es la última revisada del autor, no el pdf de la revista (esto sólo lo autorizan expresamente seis editoriales que publican seis revistas). Además suele establecerse como requisito el que en la versión depositada haya una mención explícita de la fuente donde se ha publicado el trabajo y se establezca un enlace con la revista y, en ocasiones, con la versión del artículo publicada por la editorial.

\section{Otras posibilidades de acceso abierto a los traba- jos de las revistas estudiadas}

Algunas editoriales plantean la posibilidad de que el autor (o su institución) pague cierta cantidad de dinero para que el artículo se publique en acceso abierto, sobre todo para cumplir con los requisitos que marcan algunas agencias financiadoras. Esta opción la brindan en total 11 editoriales. De ellas, 6 están clasificadas como verdes: Springer, John Wiley \& Sons (Open Choice); Elsevier (Sponsorship Option) para algunas revistas, BMJ Journal Publishing (BMJ Unlocked) y la Biophysical Society. Entre las editoriales azules sólo menciona esta posibilidad la American Society for Haematology. En el caso de las editoriales clasificadas con el color amarillo esta vía la ofrecen cuatro de las cinco: Oxford University Press (Oxford Open), Blackwell Publishing (Online Open), Nature Publishing, Taylor \& Francis (iOpenAccess). Por último, esta opción sólo la considera una editorial clasificada con el color blanco: Society for General Microbiology que lo ofrece con el nombre Open Option.

\section{Discusión}

Los datos del proyecto Romeo han demostrado ser muy útiles para conocer las posibilidades de autoarchivo, pero aunque recopila información sobre más de 8.000 revistas científicas internacionales, los datos del ámbito español están prácticamente ausentes. Esta situación puede afectar negativamente a iniciativas que incluyan entre sus objetivos el depósito de la producción científica española.

Las editoriales estudiadas establecen criterios claros y explícitos para el depósito en repositorios institucionales, mientras que no lo hacen para los temáticos. Así, hay permiso para el autoarchivo del 56,8\% de los trabajos, y si le sumamos aquellos archivables tras un período de embargo la cifra asciende al $72 \%$. El embargo es un escollo para el funcionamiento de un repositorio, pues si ya es difícil lograr el depósito de la versión del autor en el momento de aceptación del trabajo, más complicado es conseguir que los autores la depositen transcurridos seis meses de su publicación. Una forma de sortear esta dificultad es solicitando que se realice el depósito inmediato, quedando a cargo del repositorio el momento en el que ese documento puede hacerse visible. Durante el tiempo de obligado impasse puede existir información de referencia del documento y vincularla con el correo del autor responsable de la correspondencia para que los usuarios que deseen consultarlo puedan solicitar una copia. Se reproduce así el sistema de solicitud de separatas que los científicos vienen utilizando desde siempre pero ahora en versión electrónica, algo que no está afectado por las condiciones de copyright de la editorial ${ }^{15}$.

Las cifras obtenidas en este estudio demuestran viable el depósito de trabajos en un repositorio institucional, ya que ese porcentaje podría aumentar hasta el $87 \%$ si consideramos los trabajos publicados en revistas accesibles a texto completo (con o sin embargo) sobre las que no consta información en Sherpa. Por coherencia puede asumirse que las revistas que permiten el libre acceso a sus contenidos también acceden al depósito de trabajos (aunque esto debe siempre confirmarse con las editoriales), aunque otro aspecto bien distinto es qué versión es la depositable, la del autor o el pdf de la revista. La posibilidad del pdf de la editorial es interesante aunque poco frecuente, porque abre la vía del depósito efectuado por los gestores del repositorio como mecanismo para agilizar el proceso y aumentar su cobertura.

Sin embargo, hay que tener en cuenta que el éxito de un repositorio institucional no sólo depende de las condiciones de autoarchivo impuestas por las editoriales sino también de la voluntad de los investigadores pues, aunque parezca paradójico, constituyen la pieza más difícil de integrar en el sistema pese a ser sus mayores beneficiarios.

Entre los factores relacionados con la resistencia a depositar ${ }^{16,17,18}$ pueden mencionarse:

- desconocimiento de los objetivos que se persiguen con el acceso abierto y de sus procedimientos,

- falta de tiempo y medios,

- indiferencia a los posibles beneficios,

- resistencia a nuevas formas de trabajo,

así como:

- obligatoriedad para el depósito,

- miedo a la pérdida de control sobre la obra,

- temor a entrar en conflicto con el editor,

- desacuerdo con el movimiento de acceso abierto y en definitiva con la idea de compartir el trabajo.

Todos estos factores son en parte la causa de la baja participación de los autores en repositorios tan emblemáticos como PubMed Central en el que la exhaustividad en el depósito no es superior al $4 \%{ }^{19}$. Aunque hay bastantes diferencias entre repositorios y entre dis- 
ciplinas, ese porcentaje suele encontrarse alrededor del $15 \%{ }^{19}$.

Esta situación mejora drásticamente con políticas institucionales que incentivan el depósito, o con mandatos de las agencias financiadoras de la investigación que lo exijan ${ }^{19}$. Desafortunadamente en nuestro país no existe nada de esto, con lo que los esfuerzos para obtener cifras aceptables de autoarchivo deben encaminarse a establecer una intensa agenda de acciones divulgativas y educativas, a diseñar un sistema de gestión de los documentos fácilmente manejable por los autores y con el mínimo consumo de tiempo y a implantar procedimientos para agilizar la identificación y obtención de documentos depositables.

La ausencia de políticas y mandatos no sólo condiciona el éxito en el depósito de los repositorios institucionales sino que -al menos en el momento actual- determina la posible existencia de un repositorio temático. En las editoriales estudiadas lo más frecuente es que no se mencione la posibilidad del depósito en un repositorio temático (de lo que se deduce que sería necesaria la consulta previa) y cuando lo hacen, lo permiten sólo cuando es un requisito establecido por la entidad que financia la investigación. A esta condición suele añadirse la necesidad de que trascurra un período de embargo. Además cuando se hace referencia al depósito en un repositorio sobre biomedicina se hace referencia a $P u b$ Med Central, algo que no sorprende demasiado ya que junto a su mirror británico UK-PubMed Central, son los únicos existentes de estas características.

Puede concluirse que para poner en marcha un repositorio temático de biomedicina y ciencias de la salud en la Comunidad Valenciana sería necesaria la concurrencia de dos condiciones que no se dan en este momento: el consentimiento de las editoriales, y la existencia de un mandato de las agencias estatales y/o autonómicas requiriendo el depósito, por lo que la viabilidad de este tipo de repositorio en nuestro entorno está lejos de ser una realidad.

Por último cabe mencionar la utilidad que han demostrado en este trabajo los resultados de estudios métricos de producción científica para áreas geográficas y temáticas concretas. No sólo porque permiten identificar el grupo de revistas donde se publica el grueso de la producción científica potencialmente archivable, sino porque posibilitan dimensionar las consecuencias, en número de trabajos afectados, de las políticas de autoarchivo de cada editorial. Estos resultados también son de gran utilidad como estándar de referencia para posteriores estudios de exhaustividad del depósito y para ilustrar a los responsables de una institución y a los autores de los trabajos, los beneficios potenciales del depósito en términos de incremento de la visibilidad de los trabajos, sobre todo de aquellos publicados en revistas cuyo acceso está condicionado por el pago de una suscripción. Este último argumento es importante como medio para incentivar el depósito, en la medida que se acompañe de evidencias científicas que demuestran la existencia de una relación positiva entre la visibilidad obtenida gracias al acceso abierto a los textos en internet y el aumento en el número de citas recibidas $^{21,22}$.

\section{Bibliografía}

1. Schwan, A.; Brown, S. "Authors and open access publishing". En: Learned publishing, 2004, v. 17, n. 3, pp. 219-224.

2. Melero, R. "Acceso abierto a las publicaciones científicas: definición, recursos, copyright e impacto”. En: El profesional de la información, 2005, v. 14, n. 4 , pp. $255-266$

3. Hixson, C. G. Los repositorios institucionales: una oportunidad para la difusión del conocimiento. Universidad Complutense. Curso de verano 2006. Consultado en: 16-12-07.

https://scholarsbank.uoregon.edu/dspace/bitstream/1794/2998/8/Escorial_ text.pdf

4. Nottingham University. OpenDOAR-the directory of open access repositories. Consultado en: 16-12-07.

http://www.opendoar.org/

5. Universidad de Alicante. RUA. Repositorio institucional de la Universidad de Alicante. Consultado en: 16-12-07.

http://www.ua.es/rua

6. Moya-Anegón, F.; Carretero-Guerra, R.; Sánchez Malo, F.; SolísCabrera, F. (dirs.). Indicadores científicos de la producción andaluza en biomedicina y ciencias de la salud (ISI, Web of Science 2003-2004). Sevilla: Junta de Andalucía. Consejería de Salud, 2006.

7. National Institutes of Health. PubMed Central. Consultado en: 16-1207.

http://www.pubmedcentral.nih.gov

8. Ukpmc Founders Group. UK PubMed Central. Consultado en: 16-12-07. http://ukpmc.ac.uk/

9. Biblioteca Nacional de Ciencias de la Salud. Instituto de Salud Carlos III. SciELO-Scientific Electronic Library Online. Consultado en: 16-12-07. http://scielo.isciii.es/scielo.php

10. Sedic (Asociación Española de Documentación e Información). $1^{a}$ Jornada técnica de bibliotecas de ciencias de la salud en el siglo XXI: electrónicas, digitales, virtuales e híbridas. Consultado en 16-12-07.

http://www.sedic.es/gt_cienciassalud_1Jornada-Tecnica2007.htm

11. Camí, J.; Suñén, E.; Méndez-Vásquez, R. "Mapa bibliométrico de España 1994-2002: biomedicina y ciencias de la salud". En: Medicina clínica, 2005, v. 124, n. 3, pp. 93-101.

12. Abad-García, M. F.; González-Teruel, A.; Sanz-Casado, E.; Lascurain M. L.; García-Zorita, C. Producción científica de la Comunitat Valenciana en materias de biomedicina y ciencias de la salud a través de las bases de datos del Institute of Scientific Information (ISI) período 20002004. Valencia: Generalitat Valenciana, Conselleria de Sanitat, Dirección General de Ordenación, Evaluación e Investigación Sanitaria, 2007.

13. University of Nottingham. Romeo Publisher copyright policies \& selfarchiving. Consultado en: 16-12-07.

http://www.sherpa.ac.uk/romeo.php

14. Washington DC principles for free access to science. Consultado en: 12-10-07.

http://www.dcprinciples.org/

15. Hitchcock, S. Boost repository content with EPrints "Request eprint" button. Consultado en: 16-12-07.

http://www.eprints.org/news/features/request_button.php

16. Keefer, A. "Los repositorios digitales universitarios y los autores". En: Anales de documentación, 2007, v. 10, pp. 205-214. 
17. Swan, A.; Dixon, S. Open access self archiving: an author study. Cornwal, Key perspectives limited, 2005. Consultado en: 16-12-07.

http://www.keyperspectives.co.uk/openaccessarchive/reports/Open\%20Ac cess\%20II\%20(author\%20survey\%20on\%20self\%20archiving)\%202005. $p d f$

18. Rowlands, I.; Nicholas D. New journal publishing models: an international survey of senior researchers. Publishers Association and the International Association of STM Publishers, 2005. Consultado en: 16-12-07.

http://www.ucl.ac.uk/ciber/ciber_2005_survey_final.pdf

19. Suber, P. "Progress toward an OA mandate at the NIH, one more time Sparc”. En: Open access newsletter, 2007, n. 112. Consultado en: 16-1207

http://www.earlham.edu/ peters/fos/newsletter/08-02-07.htm

20. Sale, A. “A researcher's viewpoint”. En: Jacobs, N. (ed.). Open access: key strategic. Technical and economic aspects. Oxford: Chandos Publish ing, 2006. Consultado en: 16-12-07.

http://eprints.utas.edu.au/257/1/Chapter.pdf

21. Harnad, S.; Brody, T. "Comparing the impact of open access (OA) vs. non-OA articles in the same journals”. En: D-lib magazine, 2004, v. 10, n.
6. Consultado en: 16-12-07.

http://www.dlib.org/dlib/june04/harnad/06harnad.html

22. Hajjem, C.; Harnad, S.; Gingras, Y. "Ten-year cross-disciplinary comparison of the growth of open access and how it increases research citation impact". En: Journal of clinical investigation, 2005, v. 28, n. 4, pp. 39-47.

$M^{a}$ Francisca Abad-García, Aurora González-Teruel, Celeste Martínez-Catalán y Francesc Giménez-Martínez, Departamento de Historia de la Ciencia y Documentación, Facultad de Medicina, Universidad de Valencia.

abad@uv.es

abad@uv.es

celeste@uv.es

francesc.gimenez@uv.es

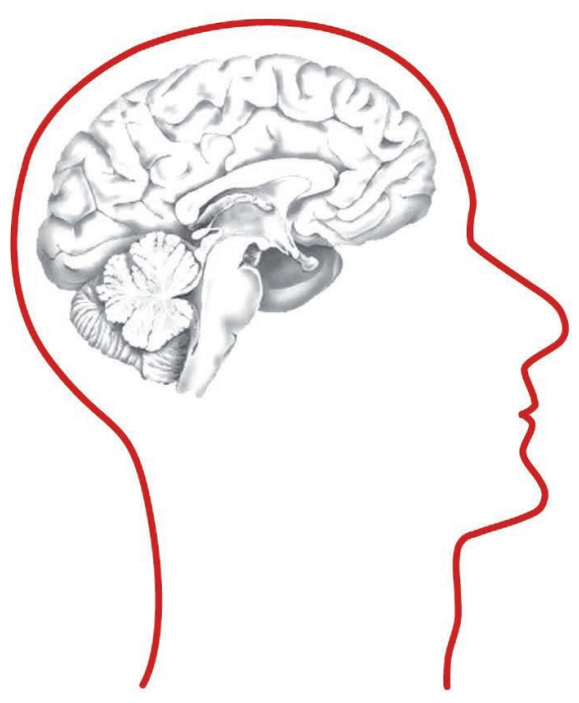

ANUARIO

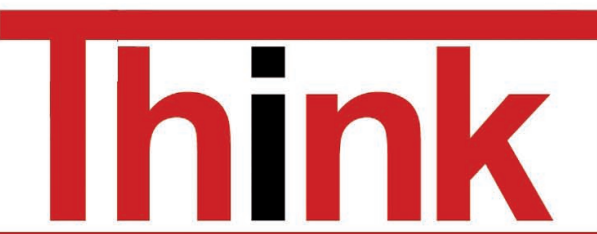

\section{Artículos en la sección GESTIÓN DE INFORMACIÓN}

Gestión de contenidos 2.0

Por Jesús Tramullas

Documentos electrónicos: Reflexiones sobre las relaciones con la tecnología de los profesionales de la información. Parte I Por Carlota Bustelo Ruesta

Documentos electrónicos: Reflexiones sobre las relaciones con la tecnología de los profesionales de la información. Parte II Por Carlota Bustelo-Ruesta

Calidad en el servicio de las bibliotecas públicas: ¿Hablamos todos de lo mismo?

Por Roser Lozano

Hemeroteca digital en la biblioteca pública

Por Javier Guallar y Ernest Abadal

Gestión de información, crisis y emergencias Por Ricardo Eito-Brun

Más información en http://www.thinkepi.net/repositorio

Ya puedes pasarnos tu pedido: epi@sarenet.es
Anuario ThinkEPI 2008

Anuario ThinkEPI $2007+2008$
$89,42 €+I V A=93 €$ $115,39 €+I V A=120 €$
Anuario ThinkEPI http://www.thinkepi.net 


\section{JORNADES CATALANES D'INFORMACIÓ I DOCUMENTACIÓ EXPGRIÈNCIA I INNOVACIÓ}

\section{BARCELONA | 22 I 23 DE MAIG DE 2008}

\section{$\{B \cdot D$}

Col-legi Oficial de Bibliotecaris-

Documentalistes de Catalunya

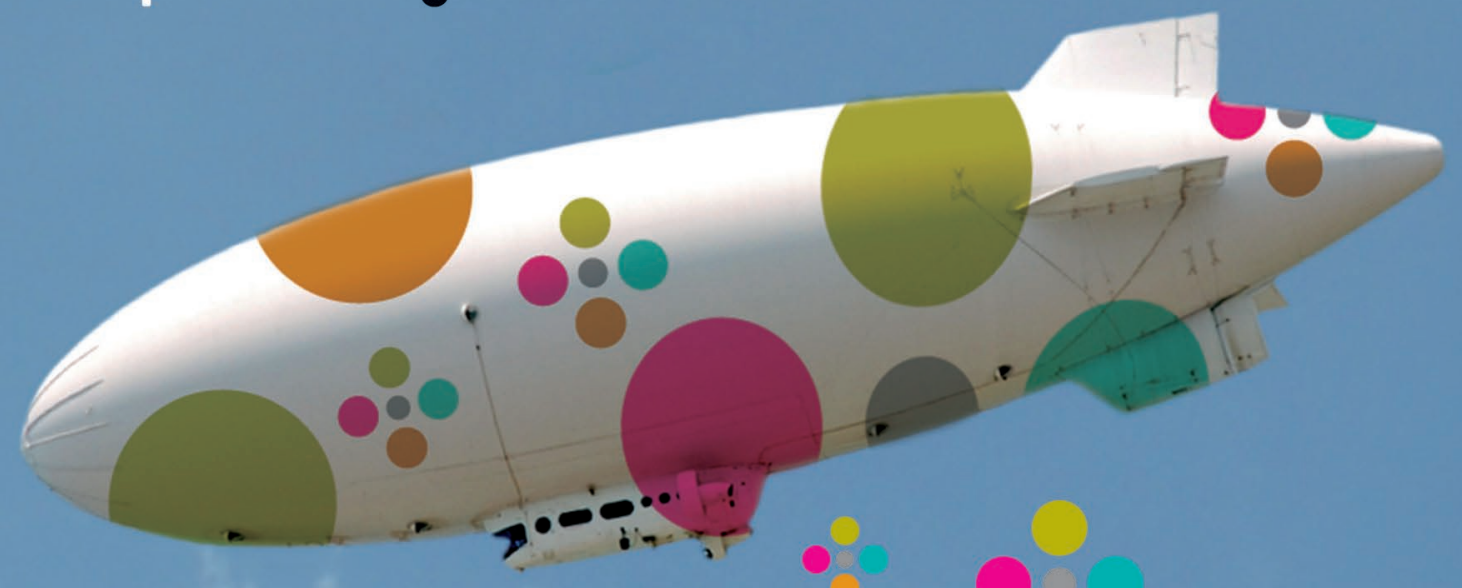

\section{NO TE LEJ POSI PERDEE!}

Més informació: http://www.cobdc.org/jornades2008 http://www.cobdc.org/jornades/11JCD/bloc/
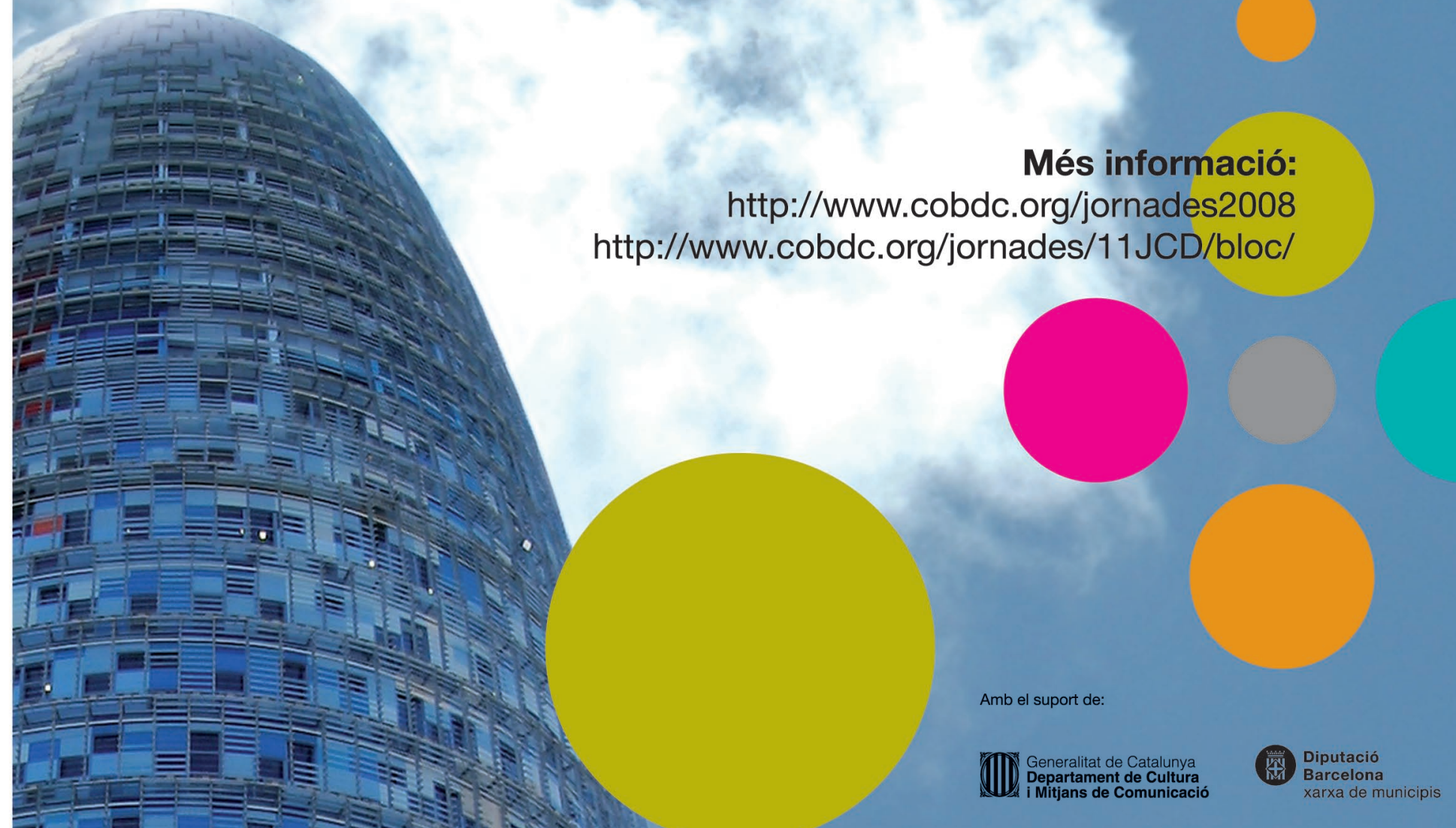